\title{
Gastrectomía en manga transumbilical con técnica simplificada: técnica y resultados quirúrgicos en 100 pacientes*
}

\author{
Drs. CARLOS FARÍAS O. ${ }^{1,2}$, CRISTIÁN OVALLE L. ${ }^{1,2}$, JOSÉ IGNACIO FERNÁNDEZ F. ${ }^{1}$, \\ JAIME DE LA MAZA C. ${ }^{1,2}$, CAROLINA CABRERA S., ${ }^{1,2}$, KARIN KOSIEL L. ${ }^{1}$, \\ ANA MARÍA MOLINA F. ${ }^{1}$, ANDRÉS MORALES T. ${ }^{1}$, Nut. ALEJANDRA REYES M. ${ }^{1}$, \\ CLAUDIA BASSO C. ${ }^{1}$, GLORIA VERA S. ${ }^{1}$, Psic. SIOMARA CHAHUAN C. ${ }^{1}$, \\ PÍA ULLOA Q. ${ }^{1}$, EDUARDO FIGUEROA M. ${ }^{1}$ \\ 1 Centro Integral de Nutrición y Obesidad, Clínica Tabancura. \\ 2 Hospital Clínico de la Fuerza Aérea de Chile. \\ Santiago, Chile.
}

\section{Abstract \\ Simplified transumbilical sleeve gastrectomy. Technique and surgical results in 100 patients}

Background: The use of transumbilical approach for sleeve gastrectomy has been recently reported, using different technique variations. Aim: To report the technique and surgical results of a transumbilical approach simplified sleeve gastrectomy, using rigid instruments. Material and Methods: Ninety four women and six men, selected by a multidisciplinary team, underwent transumbilical sleeve gastrectomy. The operative technique involved a transumbilical incision, introduction of a SILS ${ }^{\circledR}$ or GelPoint ${ }^{\circledR}$ multiport, and a $5 \mathrm{~mm}$ metallic accessory trocar laterally in the left flank. Rigid instruments were used in all patients. The greater curvature was dissected from 4-5 cm above the pylorus to the angle of His. Gastric transection was completed with a stapler, and calibrated with a 36 French tube advanced through the pylorus. Hemostasis of the staple line was carried out with metallic clips. A barium swallow was performed in ten randomly chosen patients, confirming the correct tubular shape of the stomach. Results: Body mass index of operated patients ranged from 30 to $43 \mathrm{~kg} / \mathrm{m}^{2}$. Mean operative time was $56.4 \pm 16.7$ minutes. During the early postoperative period, two patients had a hemoperitoneum, one had an antral leak and one had an intestinal perforation. No conversion to conventional laparoscopy or open technique was required. No patient died. The mean length of hospital stay was $2.3 \pm 0.5$ days. The cosmetic result was satisfactory for all patients. Conclusions: Transumbilical sleeve gastrectomy is a safe and feasible procedure with the reported technique. The insertion of an accessory $5 \mathrm{~mm}$ trocar in the left flank simplifies the procedure, allowing the use of rigid instruments.

Key words: Bariatric surgery, sleeve gastrectomy, transumbilical surgery.

* Recibido el 19 de marzo de 2012 y aceptado para publicación el 9 de abril de 2012.

Los autores no declaran conflictos de interés.

Correspondencia: Dr. José Ignacio Fernández F.

Camino El Radal 17, Condominio Los Portones de Piedra Roja, Colina, Santiago, Chile. C P: 9361721

jignaciofdez@gmail.com 


\section{Resumen}

Introducción: El abordaje transumbilical, ha demostrado ser seguro en diferentes procedimientos. Se han reportado series de casos de gastrectomía en manga transumbilical (GMTU), con diferentes variaciones. Objetivo: Es presentar la técnica y resultados quirúrgicos de una técnica de GMTU simplificada, utilizando instrumental rígido. Material y Métodos: 94 mujeres y seis hombres, fueron sometidos a GMTU, seleccionados por un equipo multidisciplinario. La técnica quirúrgica consiste en una incision transumbilical, introducción de dispositivo SILS ${ }^{\circledR}$ o GelPoint ${ }^{\circledR}$, y un trocar de $5 \mathrm{~mm}$ metálico en el flanco izquierdo. Se utilizó instrumental rígido en todos los pacientes. La disección de la curvatura mayor se realiza desde 4-5 cm proximal al píloro, hasta el pilar izquierdo. La sección gástrica se completa con stapler, calibrando con una sonda de 36 fr transpilórica. Se realiza hemostasia selectiva con clips metálicos. Se realizó estudio baritado a diez pacientes aleatorios, confirmando forma tubular adecuada. Resultados: El rango de IMC preoperatorio fue de $30-43 \mathrm{~kg} / \mathrm{m}^{2}$. El tiempo operatorio promedio fue de 56,4 $\pm 16,7 \mathrm{~min}$. No se requirió conversión a técnica multitrocar o laparotómica. Cuatro pacientes presentaron complicaciones precoces: dos hemoperitoneos, una filtración antral y una enterotomía inadvertida. Se reintervino a dos pacientes. No hubo mortalidad. El tiempo de hospitalización fue de 2,3 \pm 0,5 días. El resultado cosmético fue satisfactorio para todos los pacientes. Conclusión: La GMTU es un procedimiento factible y seguro con la técnica expuesta. La inserción del trocar de $5 \mathrm{~mm}$ accesorio, simplifica el procedimiento, permite el uso de instrumental rígido, y lo convierte en un procedimiento reproducible.

Palabras clave: Cirugía bariátrica, gastrectomía en manga, SILS, LESS, cirugía transumbilical.

\section{Introducción}

A pesar del gran salto desde la cirugía laparotómica a la cirugía laparoscópica, y sus evidentes ventajas, se ha intentado constantemente el progreso hacia procedimientos cada vez menos invasivos y con menos secuelas. Ya a principios de los años 90 se intentó reducir el número de incisiones en cirugía laparoscópica a una sola incisión, para minimizar las secuelas estéticas de procedimientos como apendicectomía ${ }^{1,2}$ y colecistectomía ${ }^{3}$; sin lograrse mayor difusión posterior de estas técnicas. En el año 2004 se da a conocer a la comunidad científica la cirugía transluminal por orificios naturales (NOTES) ${ }^{4}$; generando gran expectación y entusiasmo por los cirujanos laparoscópicos. Sin embargo, NOTES implica el riesgo de perforar vísceras huecas como forma de acceso a la cavidad abdominal, creando limitaciones tecnológicas para implementar su uso rutinario ${ }^{5}$, lo cual desvió nuevamente las miradas hacia la cirugía por puerto único. El abordaje transumbilical resultó especialmente atractivo; ya que se utiliza una cicatriz previamente existente en todos los seres humanos; la cicatriz queda prácticamente totalmente oculta, puede realizarse con instrumental laparoscópico convencional y no requiere entrenamiento adicional al que otorga la cirugía laparoscópica avanzada.

En el año 1999 es descrita por Gagner la gastrectomía en manga por vía laparoscópica (GM) como parte de la derivación biliopancreática con switch duodenal $^{6}$. Actualmente, la GM ha crecido en popularidad como procedimiento bariátrico primario, incrementándose exponencialmente el número de pacientes sometidos a este procedimiento, y a su vez las series publicadas al respecto ${ }^{7-10}$. Desde el año 2008, se han publicado diversas experiencias de gastrectomía en manga por incisión única; la mayoría de ellas en pacientes seleccionados y con tiempos quirúrgicos que habitualmente superan las dos horas $^{11-18}$. Nuestro grupo inicia en julio de $2010 \mathrm{su}$ experiencia con el abordaje transumbilical laparoscópico para GM, colecistectomía y apendicectomía. El objetivo primario de esta publicación es describir una técnica quirúrgica simplificada de GM utilizando el abordaje transumbilical (GMTU), que permita su realización con instrumental convencional, con una adecuada visibilidad y exposición, y que sea reproducible en tiempos quirúrgicos razonables.

\section{Material y Método}

Se realizó un análisis retrospectivo de la información disponible de nuestra base de datos electrónica prospectiva de cirugía bariátrica hasta el paciente número 100 en mayo de 2011. Se consideraron datos demográficos de los pacientes, y resultados quirúrgicos: tiempo operatorio, conversión a técnica mutitrocar o laparotómica, complicaciones precoces y tardías, y mortalidad. Se consideró como complicación precoz a aquella que se produjera dentro de los primeros treinta días postoperatorios, y tardía a aquella que ocurriera posterior a este período. La mortalidad se evaluó como la muerte por cualquier causa, ocurrida dentro de los treinta días postoperatorios.

\section{Pacientes}

La selección de pacientes, se realizó por nuestro equipo multidisciplinario, siguiendo las normas chi- 
lenas para el tratamiento quirúrgico de la obesidad. Se ofreció el procedimiento a todos aquellos pacientes que estuvieran en estudio para la realización de una GM; y que estuvieran interesados en un mejor resultado estético a través del abordaje transumbilical. Se consideraron como requisitos técnicos para la realización de la GMTU:

- IMC entre 30 y $43 \mathrm{~kg} / \mathrm{m}^{2}$.

- Distancia xifoumbilical menor a $25 \mathrm{~cm}$.

- Ausencia de grandes cicatrices, estrías abundantes u otros factores que anularan el beneficio estético del procedimiento.

\section{Técnica quirúrgica}

El paciente es posicionado en decúbito supino con compresión neumática intermitente. El cirujano se posiciona entre las piernas del paciente, un ayudante al costado izquierdo y otro al derecho. Se procede con la eversión y sección longitudinal del ombligo, hasta la aponeurosis (Figura 1). Se accede a cavidad peritoneal, mediante incisión de 2,5 a 3 $\mathrm{cm}$ en aponeurosis y peritoneo. Posteriormente, se introduce el sistema de puerto único. Al principio de la serie se utilizó el dispositivo SILS ${ }^{\circledR}$ (Covidien) y luego GelPoint ${ }^{\circledR}$ (Applied Medical) hasta la fecha. Bajo visión laparoscópica se introduce un trocar metálico de $5 \mathrm{~mm}$ en flanco izquierdo. Se crea neumoperitoneo a $15 \mathrm{mmHg}$. Se utiliza cámara de $5 \mathrm{~mm}$ y $30^{\circ}$ e instrumental rígido convencional. Se inicia la disección de la curvatura mayor del estómago, utilizando Enseal $^{\mathrm{TM}}$; partiendo $4-5 \mathrm{~cm}$ del píloro hasta ángulo de His. Se realiza liberación completa del fondo gástrico del pilar izquierdo. Para calibrar la gastrectomía, se posiciona a lo largo de la curvatura menor y en posición transpilórica, una sonda de 36 Fr. La sección gástrica se realiza con stapler gastrointestinales Echelon Flex ${ }^{\mathrm{TM}}$ (Ethicon Endo-Surgery), en dirección hacia el ángulo de His. Se utilizó una carga verde en la sección del antro, y cargas azules para el resto de la sección gástrica. Se realiza hemostasia selectiva de línea de corchetes con clips metálicos, sin uso de sutura. Más recientemente se ha utilizado una matriz hemostática sobre la línea de corchetes (Surgiflo®). No se realiza prueba de hermeticidad ni se instalan drenajes. La extracción del estómago resecado se realiza fácilmente a través de la incisión transumbilical; la cual posteriormente se cierra con PDS 1-0. Finalmente, se revisa a través del puerto lateral con la cámara de $5 \mathrm{~mm}$; para confirmar una correcta hemostasia de la incisión umbilical y línea de sección gástrica.

\section{Postoperatorio}

Los pacientes inician su deambulación al recuperarse de la anestesia, luego de $6 \mathrm{~h}$ de postperatorio. La ingesta de un régimen hídrico fraccionado, se inicia a las 24 h; para luego progresar a un régimen licuado a libre demanda a las $48 \mathrm{~h}$, suspendiendo todo aporte de fluidos o medicamentos endovenosos. El tercer día postoperatorio se inicia un régimen licuado. Los pacientes son dados de alta cuando logran una adecuada tolerancia oral y control de dolor con analgésicos orales; al segundo o tercer día postoperatorio.

\section{Control radiológico}

Se les realizó en forma aleatoria a 10 pacientes un control radiológico baritado; para evaluar una

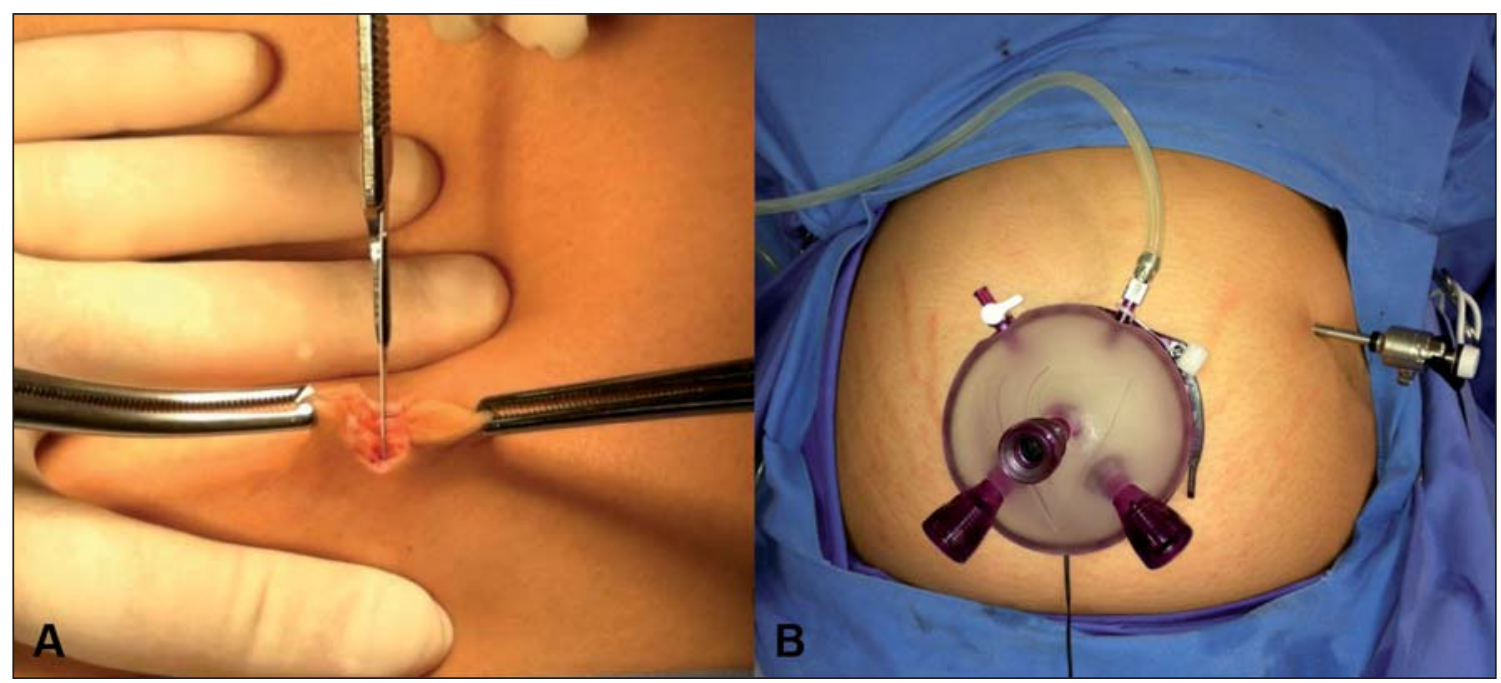

Figura 1. Detalle de acceso transumbilical simplificado. A. Incisión transumbilical. B. Dispositivo de puerto único GelPoint ${ }^{\circledR}$ y trocar accesorio de $5 \mathrm{~mm}$. 
adecuada forma del tubo gástrico. La forma considerada como adecuada para nuestro grupo, es el patrón radiológico tubular descrito por Werkin ${ }^{19}$.

\section{Resultados}

Un total de 100 pacientes fueron sometidos a GMTU, correspondientes a 94 mujeres y 6 hombres; con una edad e IMC promedio de $35 \pm 9,9$ años y $33,5 \pm 3,2 \mathrm{~kg} / \mathrm{m}^{2}$ respectivamente (Tabla 1). Las comorbilidades derivadas de la obesidad se describen en la Tabla 2. Del total de pacientes a 4 se les realizó simultáneamente una colecistectomía.

Tabla 1. Datos demográficos

\begin{tabular}{|lc|}
\hline Pacientes & $\mathbf{1 0 0}$ \\
Sexo femenino & $\mathrm{n}=94$ \\
Sexo masculino & $\mathrm{n}=6$ \\
Edad & $35 \pm 9,9$ años \\
IMC & $33,5 \pm 3,2 \mathrm{~kg} / \mathrm{m}^{2}$ \\
BMI $<35$ & $\mathrm{n}=77$ \\
BMI $35-40$ & $\mathrm{n}=19$ \\
BMI $>40$ & $\mathrm{n}=4$ \\
\hline
\end{tabular}

Tabla 2. Comorbilidades

\begin{tabular}{|l|c|}
\hline Comorbilidad & \% \\
\hline Resistencia insulina & 67 \\
\hline Dislipidemia & 49 \\
\hline Hígado graso & 48 \\
\hline RGE & 29 \\
\hline Diabetes mellitus 2 & 11 \\
\hline Osteoarticular & 10 \\
\hline HTA & 4 \\
\hline
\end{tabular}

Tabla 3. Principales complicaciones

\begin{tabular}{|l|c|}
\hline Precoces & n \\
\hline Hemoperitoneo & 2 \\
\hline Filtración & 1 \\
\hline Enterotomía inadvertida & 1 \\
\hline
\end{tabular}

El tiempo operatorio promedio para GMTU como procedimiento único fue de 56,4 $\pm 16,7$ min, y 63 min (extremos 55-70 min) con colecistectomía asociada. El promedio de estadía hospitalaria fue de $2,5 \pm 1,5$ días. No hubo conversión a técnica abierta ni multitrocar y no hubo mortalidad en este grupo.

Se observó que un $4 \%$ de los pacientes presentaron complicaciones precoces y no se registraron complicaciones tardías (Tabla 3). Fue necesario reintervenir a dos pacientes: uno por una filtración antral, debido a una falla en el cierre de la carga de stapler del segundo disparo; y otro por una enterotomía inadvertida al introducir el dispositivo SILS con una pinza Bozeman. Ambos pacientes fueron sometidos a una laparoscopia exploradora, aseo y sutura; recuperándose satisfactoriamente. Los dos pacientes que presentaron hemoperitoneo, fueron manejados médicamente; sin necesidad de reintervención. El manejo fue con aporte de volumen, suspensión de heparina de bajo peso molecular, ácido tranexámico endovenoso y aporte de fierro endovenoso.

El control radiológico aleatorio, mostró una adecuada forma tubular del tubo gástrico creado en los 10 pacientes estudiados (Figura 2). El resultado cosmético fue establecido como satisfactorio por el 100\% de los pacientes (Figura 3).

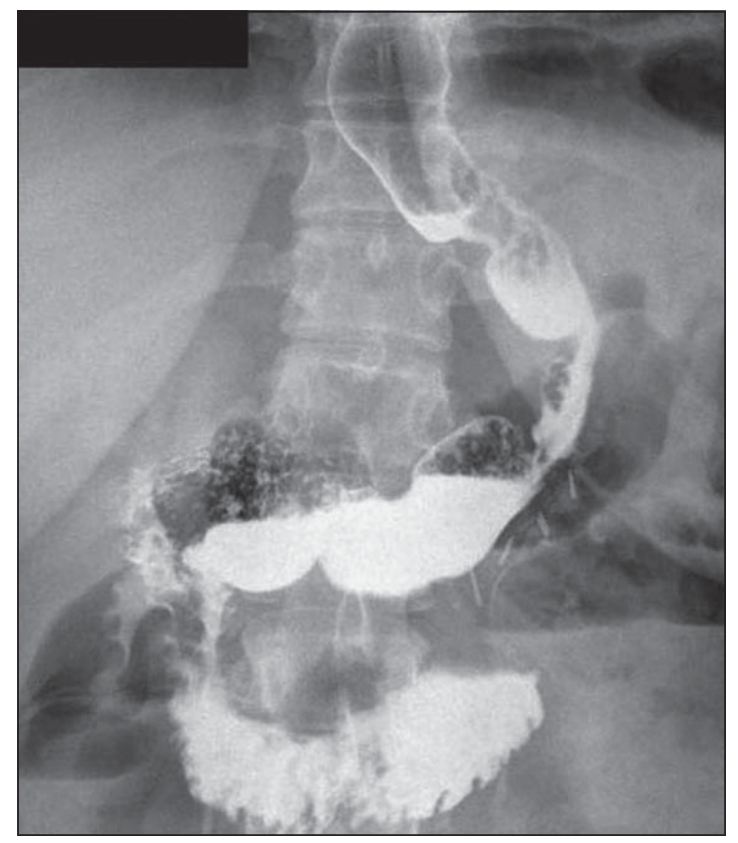

Figura 2. Estudio contrastado con bario de tubo gástrico luego de GMTU. Estudio baritado de tubo gástrico luego de GMTU. Se observa forma tubular en toda la extensión de la gastrectomía en manga, con algunas zonas de contracción peristáltica. 


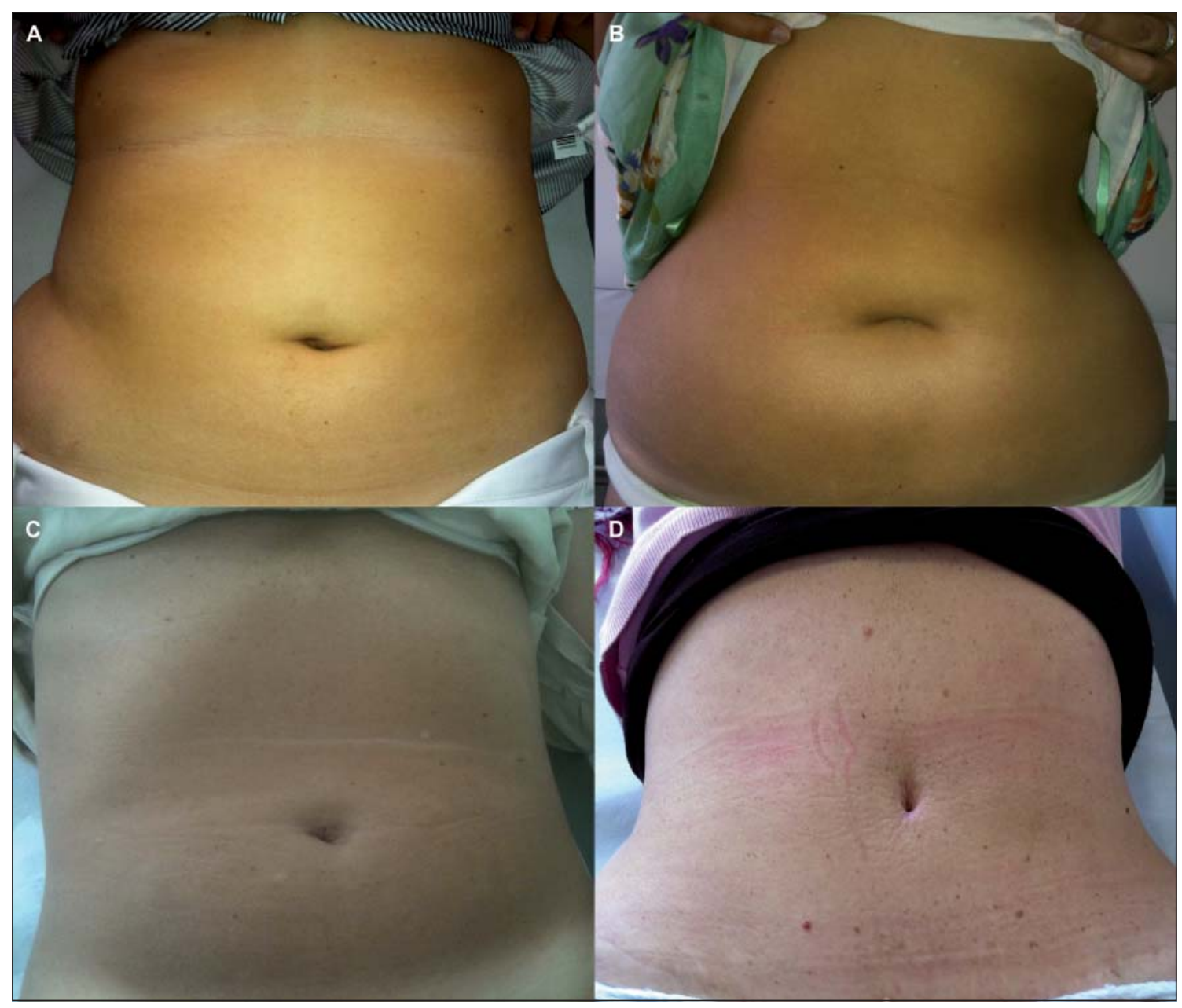

Figura 3. Resultados cosméticos de GMTU. En las imágenes se observan los resultados cosméticos de cuatro pacientes diferentes. A. Resultados 3 meses postoperatorio. B y C. Resultados 6 meses postoperatorios. D. Resultados a los 6 meses de paciente con abdominoplastía previa GMTU.

\section{Discusión}

El abordaje transumbilical en cirugía laparoscópica ha surgido como una alternativa muy atractiva, en lo que se refiere al desarrollo de técnicas de mínima invasividad. Principalmente debido a la posibilidad de realizarla de manera segura con instrumental laparoscópico convencional, cumpliendo con los objetivos de replicar la técnica quirúrgica laparoscópica multipuerto, y sin necesidad de perforar vísceras huecas.

La cirugía por puerto único, implica diversos desafíos. Los principales son la pérdida de triangulación, el conflicto de los instrumentos dentro y fuera del abdomen y disminución del número de instrumentos para tracción y contratracción. La pérdida de triangulación y el conflicto entre los instrumentos se ha manejado con la utilización de instrumental arti- culado o curvo, el cruce de instrumentos, o el uso de algún trocar accesorio de 3 ó $5 \mathrm{~mm}$. La disminución de instrumentos disponibles para tracción y contratracción, se ha podido resolver utilizando el peso o fijaciones naturales de los propios órganos intervenidos, el uso de suturas percutáneas, ganchos, etc.

La introducción de la cirugía transumbilical fue inicialmente con procedimientos relativamente sencillos como apendicectomía ${ }^{1,20,21}$ y colecistectomía ${ }^{3,22-25}$. Sus aplicaciones actuales se han extendido a un sinnúmero de procedimientos más complejos como: colectomía ${ }^{26-31}$, nefrectomía ${ }^{32-34}$, cirugía antirreflujo ${ }^{35,36}$, esplenectomía ${ }^{37,38}$, histerectomía ${ }^{39,40}$, hepatectomías ${ }^{41}$, etc. La cirugía bariátrica no ha sido ajena a estos avances, publicándose múltiples exitosas experiencias en banda gástrica ajustable $(\mathrm{BGA})^{42-44} \mathrm{y} \mathrm{GM}^{12,15,16,18}$. 
En cirugía bariátrica se agregan aún más dificultades al abordaje transumbilical. Obviamente de por si la obesidad hace más dificultoso cualquier procedimiento, principalmente aumentando la distancia xifoumbilical; lo cual implica una visión muchas veces de abajo hacia arriba y en un plano paralelo al de los órganos intervenidos ${ }^{45}$. También en estos pacientes, el hígado graso está presente frecuentemente; lo cual implica un lóbulo hepático izquierdo muchas veces de gran tamaño. El aumento de la distancia xifoumbilical, se ha manejado en diferentes experiencias básicamente seleccionando a los pacientes, utilizando instrumental de mayor longitud, o realizando la incisión para el acceso por puerto único por sobre el ombligo ${ }^{11,46}$. La separación hepática se ha solucionado a través de suturas o separadores hepáticos percutáneos, trocares accesorios o utilizando el mismo estómago como instrumento de separación ${ }^{17,47}$. A nuestro juicio, resulta imprescindible una adecuada separación hepática, para acceder y visualizar adecuadamente el ángulo de His en cirugía bariátrica; y no obtenerla constituye un riesgo no transable para el procedimiento.

La técnica expuesta utiliza el abordaje transumbilical como acceso principal para la realización de la GM y agrega un trocar accesorio de $5 \mathrm{~mm}$ en el flanco izquierdo. Si bien la técnica no corresponde a cirugía por incisión única pura; a nuestro juicio logra obtener todas las ventajas estéticas de la cirugía de puerto único, agregando una mejor triangulación de los instrumentos, y permite una adecuada visualización y exposición. Esto se traduce en tiempos quirúrgicos razonables (habitualmente bajo $60 \mathrm{~min}$ de cirugía); y versatilidad que permite realizar procedimientos asociados (como colecistectomía), sin incrementar exageradamente los tiempos quirúrgicos. Nos parece que la técnica expuesta cumple con los objetivos planteados; resultando sencilla, realizable con instrumental convencional y totalmente reproducible por cualquier cirujano entrenado en cirugía bariátrica laparoscópica.

Aunque esta serie no constituye un trabajo comparativo con la técnica laparoscópica convencional; nos parece adecuado comentar las posibles ventajas y desventajas de esta técnica. En cuanto a las ventajas, se ha sugerido en otras experiencias un mejor resultado estético, menor dolor postoperatorio y recuperación más rápida ${ }^{45}$. En nuestra experiencia, la más evidente resulta ser el resultado cosmético. Respecto al dolor postoperatorio y recuperación, no nos impresiona que existan diferencias con la técnica convencional. En relación a algunas de las posibles desventajas, estaría un mayor riesgo de hernia inicisional, dudas respecto a la seguridad del procedimiento y mayor costo. Actualmente, no existen trabajos que demuestren una mayor incidencia de hernia incisional y no es posible establecerlo con nuestra experiencia debido al poco tiempo de seguimiento. En relación a los costos del procedimiento; dado que nuestra técnica implica el uso de instrumental convencional, fuera de un leve mayor costo del dispositivo de puerto único, no hay diferencias de costo respecto a la técnica laparoscópica multitrocar. En relación a la seguridad del procedimiento; en nuestra experiencia se presentó un 4\% de complicaciones precoces. Tanto la filtración como los dos casos de hemoperitoneo, a nuestro juicio, no son atribuibles a la técnica transumbilical y su incidencia no difiere de nuestra experiencia en cirugía laparoscópica convencional. La enterotomía inadvertida, sin embargo, es claramente producto de la técnica de introducción del dispositivo de puerto único utilizado al principio de la serie; lo que llevó a cambiar el método de introducción y luego al cambio del dispositivo de puerto único.

Actualmente, nuestro grupo implementa esta técnica en forma rutinaria en aproximadamente el 90\% de las GM; y pensamos que este abordaje puede agregarse como una herramienta de uso común por otros grupos especializados en cirugía bariátrica. Resulta necesario un trabajo comparativo entre la técnica expuesta y laparoscópica convencional para establecer con mayor certeza el alcance real de las ventajas de este procedimiento.

\section{Referencias}

1. Kala Z, Hanke I, Neumann C. A modified technic in laparoscopy-assisted appendectomy-a transumbilical approach through a single port. Rozhl Chir. 1996;75:158.

2. Straslipka J. Laparoscopic appendectomy using the outtransumbilical method-personal experience. Rozhl Chir. 1997;76:85-6.

3. Bresadola F, Pasqualucci A, Donini A, Chiarandini P, Anania G, Terrosu G, et al. Elective transumbilical compared with standard laparoscopic cholecystectomy. Eur J Surg. 1999;165:29-34.

4. Kalloo AN, Singh VK, Jagannath SB, Niiyama H, Hill SL, Vaughn CA, et al. Flexible transgastric peritoneoscopy: a novel approach to diagnostic and therapeutic interventions in the peritoneal cavity. Gastrointest Endosc. 2004;60:114-7.

5. Pearl JP, Ponsky JL. Natural orifice translumenal endoscopic surgery: a critical review. J Gastrointest Surg. 2008;12:1293-300.

6. Gumbs AA, Gagner M, Dakin G, Pomp A. Sleeve gastrectomy for morbid obesity. Obes Surg. 2007;17:962-9.

7. Sarela AI, Dexter SP, O’Kane M, Menon A, McMahon MJ. Long-term follow-up after laparoscopic sleeve gastrectomy: 8-9-year results. Surg Obes Relat Dis. 2011 Jul 20. [Epub ahead of print]. 
8. Bellanger DE, Greenway FL. Laparoscopic sleeve gastrectomy, 529 cases without a leak: short-term results and technical considerations. Obes Surg. 2011;21:14650.

9. Basso N, Casella G, Rizzello M, Abbatini F, Soricelli E, Alessandri G, et al. Laparoscopic sleeve gastrectomy as first stage or definitive intent in 300 consecutive cases. Surg Endosc. 2011;25:444-9.

10. Himpens J, Dobbeleir J, Peeters G. Long-term results of laparoscopic sleeve gastrectomy for obesity. Ann Surg. 2010;252:319-24.

11. Reavis KM, Hinojosa MW, Smith BR, Nguyen NT. Single-laparoscopic incision transabdominal surgery sleeve gastrectomy. Obes Surg. 2008;18:1492-4.

12. Nguyen NT, Reavis KM, Hinojosa MW, Smith BR, Wilson SE. Laparoscopic transumbilical sleeve gastrectomy without visible abdominal scars. Surg Obes Relat Dis. 2009;5:275-7.

13. Saber AA, El-Ghazaly TH. Early experience with SILS port laparoscopic sleeve gastrectomy. Surg Laparosc Endosc Percutan Tech. 2009;19:428-30.

14. Saber AA, El-Ghazaly TH, Elian A. Single-incision transumbilical laparoscopic sleeve gastrectomy. J Laparoendosc Adv Surg Tech. 2009;19:755-8 discussion 9.

15. Varela JE. Single-site laparoscopic sleeve gastrectomy: preclinical use of a novel multi-access port device. Surg Innov. 2009;16:207-10.

16. Arias Amezquita F, Prada Ascencio NE, Gómez D, Torres A. Transumbilical sleeve gastrectomy. Obes Surg. 2010;20:232-5.

17. Galvani CA, Choh M, Gorodner MV. Single-incision sleeve gastrectomy using a novel technique for liver retraction. Jsls 2010;14:228-33.

18. Gentileschi P, Camperchioli I, Benavoli D, Lorenzo ND, Sica G, Gaspari AL. Laparoscopic single-port sleeve gastrectomy for morbid obesity: preliminary series. Surg Obes Relat Dis. 2010;6:665-9.

19. Werquin C, Caudron J, Mezghani J, Leblanc-Louvry I, Scotte M, Dacher JN, et al. Early imaging features after sleeve gastrectomy. J Radiol. 2008;89:1721-8.

20. Chouillard E, Dache A, Torcivia A, Helmy N, Ruseykin I, Gumbs A. Single-incision laparoscopic appendectomy for acute appendicitis: a preliminary experience. Surg Endosc. 2010;24:1861-5.

21. Lee J, Baek J, Kim W. Laparoscopic transumbilical single-port appendectomy: initial experience and comparison with three-port appendectomy. Surg Laparosc Endosc Percutan Tech. 2010;20:100-3.

22. Zornig C, Emmermann A, von Waldenfels HA, Mofid H. Laparoscopic cholecystectomy without visible scar: combined transvaginal and transumbilical approach. Endoscopy 2007;39:913-5.

23. Cuesta MA, Berends F, Veenhof AA. The "invisible cholecystectomy”: A transumbilical laparoscopic operation without a scar. Surg Endosc. 2008;22:1211-3.

24. Kupcsulik P, Szlavik R, Nehez L, Lukovich P. Single port transumbilical cholecystectomy [SILS] -30 nonselected cases. Magy Seb. 2011;64:69-73.

25. Qiu Z, Sun J, Pu Y, Jiang T, Cao J, Wu W. Learning curve of transumbilical single incision laparoscopic cholecystectomy (SILS): a preliminary study of 80 selected patients with benign gallbladder diseases. World J Surg. 2011;35:2092-101.

26. Bucher P, Pugin F, Morel P. Single-port access laparoscopic radical left colectomy in humans. Dis Colon Rectum 2009;52:1797-801.

27. Bucher P, Pugin F, Morel P. Transumbilical single incision laparoscopic sigmoidectomy for benign disease. Colorectal Dis. 2010;12:61-5.

28. Patel CB, Ramos-Valadez DI, Ragupathi M, Haas EM. Single incision laparoscopic-assisted right hemicolectomy: technique and application (with video). Surg Laparosc Endosc Percutan Tech. 2010;20:e146-9.

29. Rieger NA, Lam FF. Single-incision laparoscopically assisted colectomy using standard laparoscopic instrumentation. Surg Endosc. 2010;24:888-90.

30. Katsuno G, Fukunaga M, Nagakari K, Yoshikawa S, Ouchi M, Hirasaki Y. Single-incision laparoscopic colectomy for colon cancer: early experience with 31 cases. Dis Colon Rectum 2011;54:705-10.

31. Saber AA, El-Ghazaly TH. Single-incision transumbilical laparoscopic right hemicolectomy using SILS Port. Am Surg. 2011;77:252-3.

32. Zeltser IS, Bergs R, Fernández R, Baker L, Eberhart R, Cadeddu JA. Single trocar laparoscopic nephrectomy using magnetic anchoring and guidance system in the porcine model. J Urol. 2007;178:288-91.

33. Gill IS, Canes D, Aron M, Haber GP, Goldfarb DA, Flechner S, et al. Single port transumbilical (E-NOTES) donor nephrectomy. J Urol. 2008;180:637-41; discussion 41.

34. Aminsharifi A, Taddayun A, Shakeri S, Hashemi M, Abdi M. Hybrid natural orifice transluminal endoscopic surgery for nephrectomy with standard laparoscopic instruments: experience in a canine model. J Endourol. 2009;23:1985-9.

35. Hamzaoglu I, Karahasanoglu T, Aytac E, Karatas A, Baca B. Transumbilical totally laparoscopic singleport Nissen fundoplication: a new method of liver retraction: the Istanbul technique. J Gastrointest Surg. 2010;14:1035-9.

36. Dapri G, Bruyns J, Himpens J, Cadiere GB. Single-access transumbilical laparoscopic Nissen fundoplication performed with new curved reusable instruments. Surg Innov. 2011;18:61-5.

37. Targarona EM, Balague C, Martínez C, Pallares L, Estalella L, Trias M. Single-port access: a feasible alternative to conventional laparoscopic splenectomy. Surg Innov. 2009;16:348-52.

38. Oyama K, Sasaki A, Chiba T, Nitta H, Otsuka K, Wakabayashi G. Single-incision laparoscopic splenectomy for idiopathic thrombocytopenic purpura: report of a case. 


\section{GASTRECTOMÍA EN MANGA TRANSUMBILICAL CON TÉCNICA SIMPLIFICADA: TÉCNICA Y RESULTADOS ...}

Surg Today 2011;41:1091-4.

39. Jung YW, Kim YT, Lee DW, Hwang YI, Nam EJ, Kim $\mathrm{JH}$, et al. The feasibility of scarless single-port transumbilical total laparoscopic hysterectomy: initial clinical experience. Surg Endosc. 2010;24:1686-92.

40. Yim GW, Jung YW, Paek J, Lee SH, Kwon HY, Nam EJ, et al. Transumbilical single-port access versus conventional total laparoscopic hysterectomy: surgical outcomes. Am J Obstet Gynecol. 2010;203:26 e1-6.

41. Zhao G, Hu M, Liu R, Xu D, Ouyang C, Xu Y, et al. Laparoendoscopic single-site liver resection: a preliminary report of 12 cases. Surg Endosc. 2011;25:3286-93.

42. de la Torre RA, Satgunam S, Morales MP, Dwyer CL, Scott JS. Transumbilical single-port laparoscopic adjustable gastric band placement with liver suture retractor. Obes Surg. 2009;19:1707-10.

43. Saber AA, El-Ghazaly TH. Early experience with single incision transumbilical laparoscopic adjustable gastric banding using the SILS Port. Int J Surg. 2009;7:456-9.

44. Teixeira J, McGill K, Koshy N, McGinty J, Todd G. Laparoscopic single-site surgery for placement of adjustable gastric band-a series of 22 cases. Surg Obes Relat Dis. 2010;6:41-5.

45. Saber AA, El-Ghazaly TH, Dewoolkar AV, Slayton SA. Single-incision laparoscopic sleeve gastrectomy versus conventional multiport laparoscopic sleeve gastrectomy: technical considerations and strategic modifications. Surg Obes Relat Dis. 2010;6:658-64.

46. Saber AA, El-Ghazaly TH. Feasibility of single-access laparoscopic sleeve gastrectomy in super-super obese patients. Surg Innov. 2010;17:36-40.

47. Saber AA, Elgamal MH, Itawi EA, Rao AJ. Single incision laparoscopic sleeve gastrectomy (SILS): a novel technique. Obes Surg. 2008;18:1338-42. 